\title{
Transitioning to online instruction: Strong ties and anxiety
}

\author{
Eric Brewe $\odot,{ }^{1}$ Adrienne Traxler $\odot,{ }^{2}$ and Sarah Scanlin $\oplus^{3}$ \\ ${ }^{1}$ Drexel University, Department of Physics, School of Education, Philadelphia, Pennsylvania 19104, USA \\ ${ }^{2}$ Wright State University, Department of Physics, Dayton, Ohio 45435, USA \\ ${ }^{3}$ Drexel University, Department of Electrical and Computer Engineering, \\ Philadelphia, Pennsylvania 19104, USA
}

(Received 4 February 2021; revised 27 April 2021; accepted 28 June 2021; published 22 July 2021)

\begin{abstract}
We surveyed a national sample of United States physics faculty about the COVID-19 transition to online learning. Most faculty had 1-2 weeks to prepare and no experience with teaching online. They relied on department peers to discuss approaches and used lecture adaptations such as video conferencing rather than new curricular elements. Their responses were empathetic to the students' situation, and $90 \%$ believed they were average or above at implementing online instruction. Faculty's preference for local resources and existing methods suggests that in a crisis, strong network ties will dominate as information sources, with consequences for professional development and instructional change.
\end{abstract}

DOI: 10.1103/PhysRevPhysEducRes.17.023103

\section{INTRODUCTION}

The transition to online instruction brought on by COVID-19 forced faculty who by and large did not have experience with online teaching to adapt courses rapidly to online instruction. This transition required faculty to identify and implement new approaches to delivering instruction and assessing students. Lewin's model of organizational change includes three phases: unfreezing, movement, and refreezing [1]. The changes brought about by COVID-19 represent an unfreezing of instructional practice. COVID-19 has been a public health emergency, and simultaneously has required faculty to adapt to new instructional conditions-often during times of personal crisis. Because it forced changes in instruction, this unfreezing was also a potential time to take up existing research-based course materials that were suitable for online instruction. In this case, disruption happened quickly and under high-stress circumstances, which may have reinforced entrenched practices and required faculty to draw on local resources for information and strategies.

One of the differences between the COVID-19 transition and established theories of instructional change [2] is that research on instructional change tends to emphasize intentional changes, made deliberately over time and with planning $[3,4]$. In contrast, the changes precipitated by the pandemic were largely involuntary and made with little planning time. A prevalent view on instructional change,

Published by the American Physical Society under the terms of the Creative Commons Attribution 4.0 International license. Further distribution of this work must maintain attribution to the author(s) and the published article's title, journal citation, and DOI. commonly known as develop and disseminate, holds that when faculty need new instructional materials or desire to change instructional practice, they will seek out and implement established curricula. This view is common among discipline-based education researchers, who have spent considerable time developing and researching curriculum materials and promoting their adoption [5]. However, it is often not shared by faculty practitioners, who may find these materials inflexible or impractical to implement [6,7]. During the spring and summer of 2020, groups such as the American Association of Physics Teachers and PhysPort published and widely emailed guides highlighting key research-based instructional resources. The "develop and disseminate" framework would suggest that the internet and online social networks were key sources for ideas about online teaching, as inperson venues such as conferences disappeared.

To probe what happened in this transition, we surveyed physics faculty about their moves to online learning. This paper summarizes the quantitative results from that survey; future work will discuss the often lengthy and nuanced text responses.

\section{METHODS}

In order to characterize instructional decision making as faculty transitioned to online instruction, we developed a survey that focuses on faculty's approaches, information sources, and efficacy beliefs around the transition. We selected faculty at the 10 largest colleges and universities in each of the 50 states as well as the District of Columbia, Puerto Rico and the U.S. Virgin Islands (20 largest in California, Texas, Florida, New York, and Pennsylvania), identified by the site College Navigator. The dataset was 
restricted to two- and four-year, public or private, not-forprofit schools. After checking that each school had transitioned to online instruction, the physics department website was scraped for faculty emails. This list of roughly 600 schools yielded approximately 14000 faculty emails. The survey was distributed between May 18-July 7, 2020 using Qualtrics. No incentives were offered to faculty for their participation. There were 1057 unique responses. Responses included 49 states plus the District of Columbia and the Virgin Islands and over 200 distinct schools. Of these, 718 respondents taught physics during the spring 2020 COVID-19 transition to online teaching. We filtered to those who finished more than $50 \%$ of the survey; these 662 respondents are the subset of the data analyzed further.

The survey had 38 questions split into broad categories: Characteristics of institutions and classes transitioned online (7), Sources of information about online teaching (8), Tools and techniques implemented (5), Self efficacy beliefs about online teaching (5), Sources of anxiety in online and in person teaching (7), Perceived stability of job (3), Demographics (2), and Open-ended (1). Self efficacy questions were adapted from the Ohio State Teaching Efficacy Scale [8] and used a five-point Likert scale. Questions about online teaching were adapted from the Mathematics Teaching Anxiety Scale [9] and used a four-point Likert scale.

Respondents were asked for gender identity and racial or ethnic background. Four hundred and sixty-seven participants $(71 \%)$ identified as male, $147(22 \%)$ as female, $7(1.1 \%)$ as a nonbinary gender option, and $19(2.9 \%)$ preferred not to say. Four hundred and ninety-two respondents $(74 \%)$ indicated they were White; 65 (9.8\%) Asian, 28 (4.2\%) Hispanic, Latina/o, or of Spanish origin; 8 (1.2\%) Black or African American; 7 (1.1\%) Middle Eastern or North African; 2 American Indian or Alaska Native, and 2 Native Hawaiian or Pacific Islander. Fifteen $(2.3 \%)$ indicated "Other" on the race and ethnicity question, and 35 (5.3\%) preferred not to answer. For both questions, participants could select all that applied or could skip the question, leading to totals other than $100 \%$ in some cases. These percentages are similar to recent American Institute of Physics (AIP) statistics on gender [10] and race [11] of physics faculty, though they cannot be exactly compared because the AIP reports binary gender and fewer race and ethnicity categories.

\section{A. Data handling}

The descriptive data (demographics, institutional data, experience) included complete cases for 656 of the 662 responses. For the data regarding changes to instruction we have complete cases for 643 of 662 responses. Questions on efficacy and anxiety were used in modeling. These include 637 complete cases which represents $96 \%$ of the total responses. We used multiple imputation to develop five complete datasets using predictive mean modeling in the MICE package in R [12]. Because less than 5\% of these data were missing, we chose to omit data row-wise without imputation, and upon comparing results between the imputed dataset and the complete cases analysis we did not see differences between results. The anxiety questions included repeated questions that probed anxiety in both face-to-face and online instruction (e.g., "I would be anxious if my dean observed my face-to-face class" and "I would be anxious if my dean observed my online class"). There were three pairs of anxiety questions, so the responses were aggregated into online and face-to-face anxiety scales by summing the responses and then dividing by 12 (the maximum). All data were analyzed using R [13] and RStudio [14]. Missing data were omitted row-wise.

\section{B. Data analysis}

The efficacy and anxiety questions were analyzed using a Bayes factor approach. Bayes factors are similar to the $p$ value in frequentist statistics in that Bayes factors quantify the strength of the belief in a model as compared to a null model. The Bayes factor is based in an odds ratio of the posterior distributions from a model and the null. This makes the Bayes factor easily interpretable: a ratio of $3: 1$ indicates that the evidence is 3 times as strong in favor of one model over the other, ratios around 1:1 indicate that neither model is preferred, and ratios less than 1 indicate that the null model is preferred [15]. Further, Bayes factors are invertible-meaning that we can compare the strength of belief in the null model. Our analyses of efficacy beliefs all use the BayesFactor package in R [16]. For the efficacy questions, we used the Bayesian test of association, which is the Bayesian analog to a $\chi^{2}$ test. We then took the inverse to find the evidence in favor of the null model which indicates no association. For the anxiety questions, we used the Bayesian $t$ test. Bayes factors are a useful approach in this case as we wanted to establish the strength of belief in the null model.

\section{RESULTS}

\section{A. Resources and tools used}

As faculty moved courses to online instruction, they reported drawing most frequently on other faculty in their own departments. Further, respondents found their colleagues to be among the most useful resources for information about online instruction. As shown in Fig. 1, the dominant resource was faculty within the same department, and three of the top five categories involved talking with other faculty (four of the top five, depending on the faculty composition of the local teaching center). In a ranking of most productive resources, the three faculty categories [others in my department (1.90), physics colleagues in other institutions (2.64), and faculty in other departments (2.94)] all ranked in the top four. Campus teaching and learning centers, including science, technology, engineering, and 

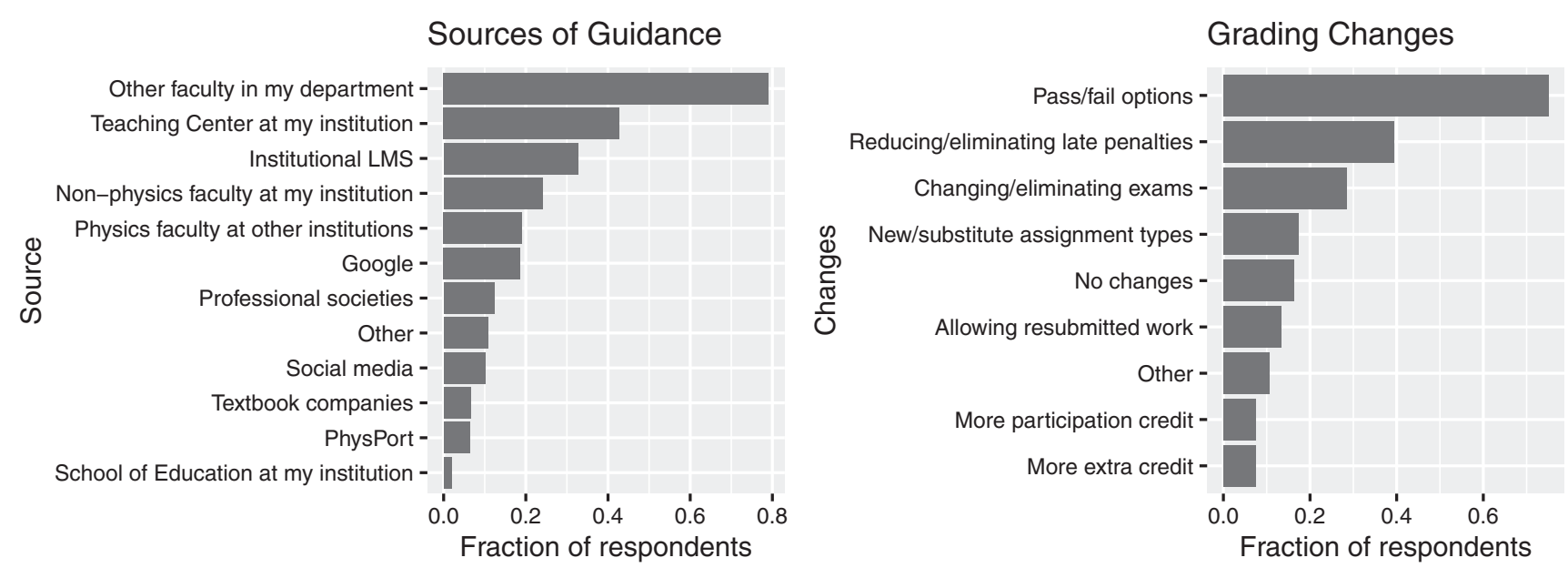

FIG. 1. Resources used by faculty. Left: Sources of guidance used in the transition to online instruction. "Social media" includes Twitter and Facebook. "Professional societies" includes the American Association of Physics Teachers and the American Physical Society. Right: Most commonly chosen changes to course structure.

mathematics focused centers, were both highly used and seen as productive resources. Generally, faculty reported no experience with online teaching (77\%)—-though faculty in non-Ph.D. granting departments had greater levels of online experience, see Fig. 2.

Seeking local resources for information was precipitated by the lack of time for planning, with $71 \%$ of faculty having between 5 days and 2 weeks to prepare. Effectively, for most this was a survival mode. When asked about new tools used in their suddenly-online courses, faculty primarily reported video conferencing $(89 \%)$ and features available within their institutional Learning Management System (42\%). The disruption presented by online instruction did not motivate much use of novel techniques or tools; less than $25 \%$ of respondents reported using online whiteboards, digital

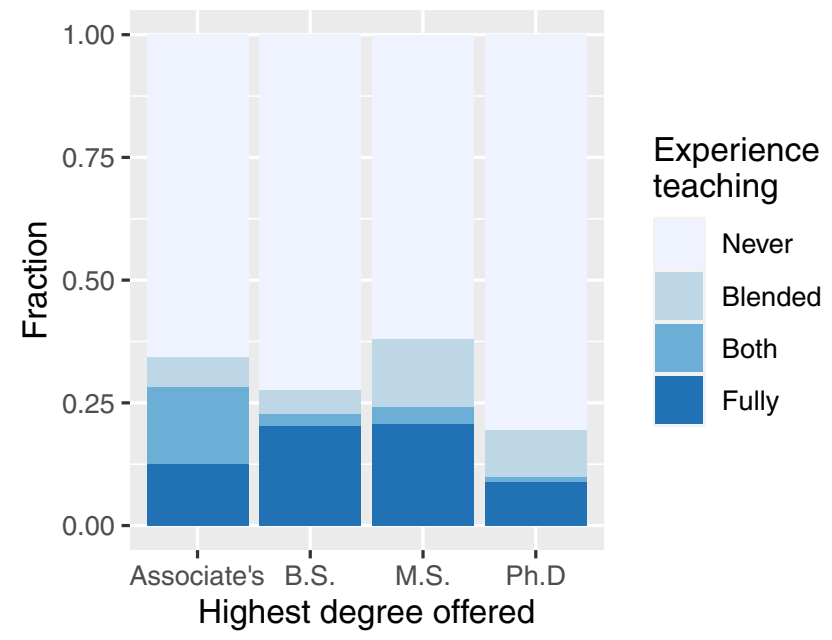

FIG. 2. Prevalence of online teaching experience by highest degree offered in department: associate's degree (32), bachelor's degree (123), master's degree (29), and Ph.D. (477). polling, or online simulations or demonstrations - which is in line with reported levels of active learning [17]. This indicates existing approaches, like lecturing, became further entrenched by becoming part of the architecture of online classes.

\section{B. Assessment, anxiety, and efficacy}

While the mode of instruction may have stayed consistent in the shift to online instruction, respondents reported substantial changes to assessment strategies, evaluation criteria, and class practices. As shown on Fig. 1, only $17 \%$ of respondents reported making no changes to their class, content covered, or modes of assessment. Three quarters of the respondents indicated that pass or fail options were added to the grading scheme. This was typically an institution-level decision, but several other faculty-controlled changes were also common, including reducing or eliminating penalties for late work (40\%), changing or eliminating exams (29\%), using new or substitute assignment types (17\%), allowing students to resubmit work (13\%), and increased participation credit or extra credit opportunities (7\% each).

As seen in Fig. 3, we found that faculty are more anxious about teaching online than in face-to-face settings. A Bayes factor paired samples $t$ test found evidence for greater anxiety in online settings as compared to face to face settings (Bayes factor: $2.9 \times 10^{11}: 1$ ). We note that the transition to online teaching is also coincident with a global pandemic and a lack of experience with online instruction, so the generalizability of this result is limited.

Despite these concerns, over $90 \%$ of respondents rated their online instruction as average or above when responding to the question "How well can you implement online instructional strategies for your students?"; see Fig. 4. To explore how other variables related to efficacy beliefs, we 


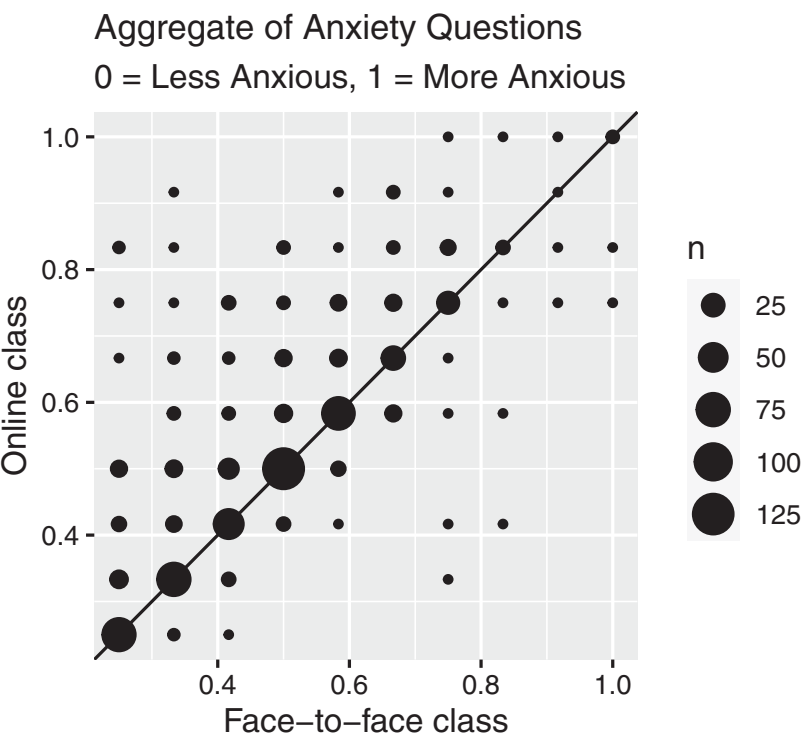

FIG. 3. Faculty ranking of aggregate anxiety comparing online vs face-to-face instruction.

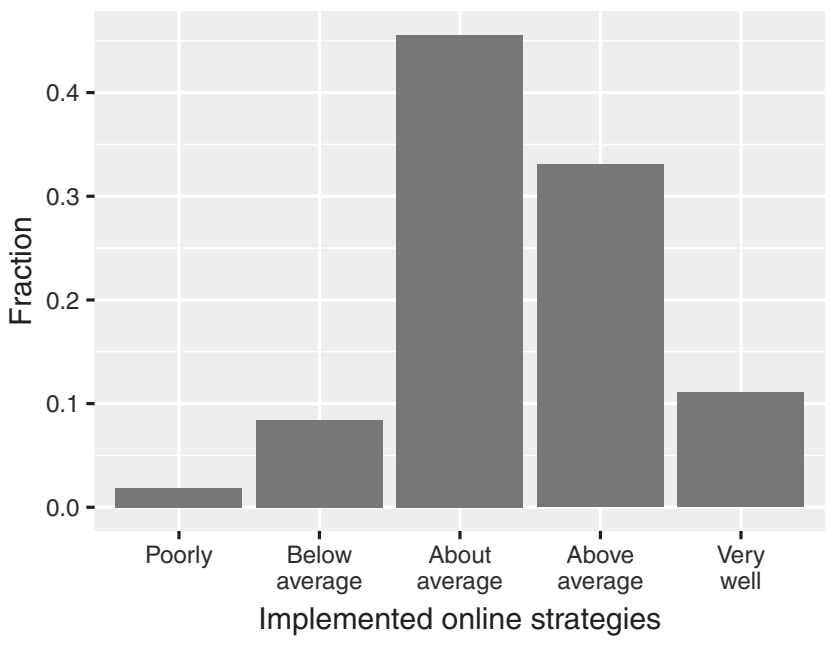

FIG. 4. Perceived efficacy of implementing online instruction.

conducted Bayesian analyses of association with gender (for participants who identified as "female" or "male"), level of course transitioned, institution type, time to implement, perceived job security, experience with online instruction, or faculty rank. The tests of association produced Bayes factors indicating strong evidence for the null model (no association between variables) as seen in Table I for all variables except "Experience with online instruction." The null model was only moderately favored $2.7: 1$ by the Bayes factor for this variable. Kass and Raftery [18] describe this as evidence "worth no more than a bare mention." Many faculty in our sample were from Ph.D.-granting departments, and those faculty typically taught fewer courses. To see if faculty with lighter teaching loads were more confident about their effectiveness, we
TABLE I. Bayes factors for tests of no association between efficacy and other potentially predictive variables. There was no association with male or female gender, faculty rank, job security, course level, institution type, implementation time, or experience with online instruction.

\begin{tabular}{lc}
\hline \hline Variable & Bayes factor \\
\hline Gender (dichotomized) & 121 \\
Course level & 4390 \\
Institution type & $1.24 \times 10^{9}$ \\
Time to implement & $1.31 \times 10^{7}$ \\
Job security & $6.90 \times 10^{5}$ \\
Experience with online instruction & 2.70 \\
Faculty rank & $1.99 \times 10^{4}$ \\
\hline \hline
\end{tabular}

separated Fig. 4 by typical number of courses taught, and found no difference.

\section{DISCUSSION}

Our results reflect the challenges of a rapid transition from face-to-face to online instruction in physics. In order to cope with this transition, faculty drew heavily on local resources such as colleagues and teaching and learning centers within their own institutions. Despite the plethora of advice and resources available through the global community connected by social media, we found that very few faculty used Twitter or Facebook as a source of information in this transition (5\% each). Instead, they preferred local resources who would understand contextual factors such as the LMS and the availability of resources for doing online instruction. The focus on local sources is consistent with work done on information sources for research based instruction [19] and prevailed even when in-person meetings and "hallway chat" were no longer an option. In a network sense, these physically proximate and high-frequency contacts were most likely to be strong ties [20], as opposed to the diffuse acquaintances promoted by Twitter or Facebook.

An open-response survey item asked faculty about their experiences with teaching online, how they transitioned, and lessons learned. The response was voluminous, and analysis of those data is ongoing. Concern for students was a recurring theme, though it was not explicitly part of the prompt. Many faculty mentioned that students tended to keep video off in synchronous sessions, making it difficult for faculty to connect with them or estimate the level of class engagement. Others mentioned the lack of class community or cohesion, or commented on student mood (e.g., "everyone was in shock"). Faculty were also reflective and concerned about tradeoffs in their instructional decisions: for example, keeping classes synchronous exacerbated gaps in internet access, but asynchronous instruction shifted a much higher burden of scheduling and self-structure to students. 


\section{IMPLICATIONS AND FUTURE WORK}

Faculty did not use the disruption caused by COVID-19 to implement novel instructional approaches, and instead primarily focused on making lectures available through video conferencing software and their learning management systems. Because lecture is the prevalent instructional mode [17], the translation to online instruction may further entrench lecturing as a practice. This may be heightened by limiting the sources of guidance to local resources rather than professional societies or organizations that specialize in providing professional development.

Yet, faculty did make instructional changes. These changes can be seen as empathetic, adjusting grading and success criteria to respond to the myriad anxieties and complexities of instructional decision making. Many faculty adaptations to their unfamiliar instructional setting appear to have focused on supporting students and offering multiple ways to succeed in the crisis. These themes emerged most strongly in the open response and interview data, with details forthcoming in a future paper. Faculty developers and professional societies who hope to improve educational systems should attend to this faculty concern for students. It provides an opening to motivate more substantial curricular changes-if faculty have the time and resources to make such reforms feasible.

The perceived positive experience with implementing online instruction (Fig. 4) is interesting, because instructional changes require significant cognitive resources and time [21]. None of the variables we explored were associated with different rankings of efficacy, which is surprising, as it implies that neither resources nor experience nor demographic factors benefit faculty in terms of their efficacy beliefs. One interpretation of this result is that faculty were respectful of their own efforts in the face of a challenging instructional situation. Also, the subset of faculty who responded to the survey may skew toward those who are motivated to invest time and energy in teaching (though that does not necessarily translate into self-efficacy).

As faculty implement online instruction during the 2020-2021 academic year, they may continue to rely on strong ties, or they might reach beyond their local contexts for best practices and guidance from curriculum or online instruction experts. Gathering follow-up data about faculty practices in this time can give key information about where funding agencies should invest in educational reform. If the short-term nature of the crisis was the primary driver of preferring local sources, then faculty would be expected to report a greater range of resources and practices in the fall 2020 and spring 2021 terms. However, if strong network ties are the driving mechanism, then instructors will continue to rely on local contacts and existing practices. In this scenario, professional development that builds strong community ties may be one of the only routes for research-based educational change to succeed [22].

\section{ACKNOWLEDGMENTS}

The Institutional Review Board of Drexel University approved this study. Informed consent was obtained from all participants. This project was supported by NSF DUE 2027958 and DUE 2027963. We are grateful to all the faculty who took time to respond to the survey in the midst of an overwhelming time. E. B. and A. T. contributed equally to this work and should be considered co-first authors.
[1] K. Lewin, Frontiers in group dynamics: Concept, method and reality in social science; social equilibria and social change, Human Relat. 1, 5 (1947).

[2] M. Borrego and C. Henderson, Increasing the use of evidence-based teaching in STEM higher education: A comparison of eight change strategies, J. Eng. Educ. 103, 220 (2014).

[3] M. Dancy and C. Henderson, Pedagogical practices and instructional change of physics faculty, Am. J. Phys. 78, 1056 (2010).

[4] C. Henderson, A. Beach, and N. Finkelstein, Facilitating change in undergraduate STEM instructional practices: An analytic review of the literature, J. Res. Sci. Teach. 48, 952 (2011).

[5] C. Henderson, N. Finkelstein, and A. Beach, Beyond dissemination in college science teaching: An introduction to four core change strategies, J. Coll. Sci. Teach. 39, 18 (2010), https://eric.ed.gov/?id=EJ887499.
[6] C. Henderson and M.H. Dancy, Physics faculty and educational researchers: Divergent expectations as barriers to the diffusion of innovations, Am. J. Phys. 76, 79 (2008).

[7] C. Henderson, M. Dancy, and M. Niewiadomska-Bugaj, Use of research-based instructional strategies in introductory physics: Where do faculty leave the innovationdecision process?, Phys. Rev. ST Phys. Educ. Res. 8, 020104 (2012).

[8] M. Tschannen-Moran and A. W. Hoy, Teacher efficacy: Capturing an elusive construct, Teach. Teach. Educ. 17, 783 (2001).

[9] M. Peker, Mathematics teaching anxiety and self-efficacy beliefs toward mathematics teaching: A path analysis, Educ. Res. Rev. 11, 97 (2016).

[10] C. Helba, A. M. Porter, S. Nicholson, and R. Ivie, Women among physics and astronomy faculty, Tech. Rep. (American Institute of Physics, College Park, MD, 2019). 
[11] R. Ivie, G. Anderson, and S. White, African Americans \& Hispanics among physics \& astronomy faculty, Tech. Rep. (American Institute of Physics, College Park, MD, 2014).

[12] S. van Buuren and K. Groothuis-Oudshoorn, mice: Multivariate imputation by chained equations in R, J. Stat. Softw. 45, 1 (2011).

[13] R Core Team, R: A Language and Environment for Statistical Computing, R Foundation for Statistical Computing, Vienna, Austria (2017).

[14] RStudio Team, RStudio: Integrated Development Environment for $R$ (RStudio, PBC., Boston, MA, 2020).

[15] D. Navarro, Learning statistics with R: A tutorial for psychology students and other beginners (2013) pp. 1542, https://learningstatisticswithr.com/.

[16] R. D. Morey and J. N. Rouder, BayesFactor: Computation of Bayes Factors for Common Designs (2018), R package version 0.9.12-4.2.

[17] M. Stains et al., Anatomy of STEM teaching in North American universities, Science 359, 1468 (2018).
[18] R. E. Kass and A. E. Raftery, Bayes factors, J. Am. Stat. Assoc. 90, 773 (1995).

[19] M. Dancy, C. Henderson, and C. Turpen, How faculty learn about and implement research-based instructional strategies: The case of Peer Instruction, Phys. Rev. Phys. Educ. Res. 12, 010110 (2016).

[20] D. Krackhardt, The strength of strong ties: The importance of philos in organizations, in Networks in the Knowledge Economy, edited by R. Cross, A. Parker, and L. Sasson (Oxford University Press, New York, 2003), pp. 216-239.

[21] C. Henderson, The challenges of instructional change under the best of circumstances: A case study of one college physics instructor, Am. J. Phys. 73, 778 (2005).

[22] K. Quardokus and C. Henderson, Promoting instructional change: Using social network analysis to understand the informal structure of academic departments, Higher Educ. 70, 315 (2015). 